\title{
Analysis of the Performance of an Open-Cathode Polymer Electrolyte Fuel Cell Stack Using Simultaneous Electrochemical Impedance Spectroscopy Measurements
}

\author{
Samuel Cruz-Manzo, Rui Chen and Paul Greenwood \\ Department of Aeronautical and Automotive Engineering, Loughborough University, \\ Leicestershire, LE11 3TU, UK
}

\begin{abstract}
In this study, an analysis of the performance in a commercial opencathode polymer electrolyte fuel cell (PEFC) stack is conducted using simultaneous electrochemical impedance spectroscopy (EIS) measurements. The factors that limit the performance of the PEFC stack were evaluated with EIS measurements and the impedance model developed in the authors' previous study. The results showed that adsorbed intermediate species during the oxygen reduction reaction (ORR) limit the PEFC stack performance. The activation overpotential in the stack was dominated by low electrocatalytic activity from one of the cells. The inhomogeneity of air flow rate for this particular PEFC stack played an important role in oxygen transport limitations and ohmic resistance. This study has demonstrated that simultaneous EIS is a powerful tool for in-situ diagnosis of a PEFC stack. This EIS understanding has enabled an assessment of the state of health and performance of the fuel cell stack.
\end{abstract}

\section{Introduction}

Polymer electrolyte fuel cells (PEFCs) generate electrical and thermal energy by combining hydrogen and oxygen. Open-cathode PEFCs have to survive under a range of operational environments varying from, for example, a winter low of sub-zero air temperatures to a summer high relative humidity. They also have to survive under a range of atmospheric compositions which can include sulphur dioxide, nitrogen oxides and ionic contamination, as well as fuel impurities that can all potentially cause irreversible damage to the PEFC (1). The list of critical contaminants is much longer if we also consider the effects of battlefield gases for military applications (2). Electrochemical impedance spectroscopy (EIS) is a powerful technique that can be applied in-situ for diagnosis of the PEFC performance. The resulting impedance is commonly shown in a complex plane and represents the electrochemical and diffusion mechanisms in the frequency domain. In this study an analysis of the performance of an open-cathode PEFC stack using simultaneous EIS measurements has been carried out. The objective of this study is to provide an insight into the change in impedance of individual cells within a commercial open-cathode 4-cell stack. The electrochemical and diffusion mechanisms in each cell are calculated through the theoretical treatment developed in previous studies $(3,4)$; therefore it is possible to have an insight into the factors that limit the performance of the PEFC stack. 


\section{Experimental EIS Measurements}

A commercial open-cathode 4-cell stack with a $16 \mathrm{~cm}^{2}$ area was used for the experimental tests. The membrane electrode assemblies (MEAs) were made of Nafion 211 with platinum loadings of $0.4 \mathrm{mg} / \mathrm{cm}^{2}$ and carbon black for the electrodes. The gas diffusion layers (GDLs) were made of carbon felt with $200 \mu \mathrm{m}$ width. The 4-cell stack consists of open cathodes with two 5V DC fans for oxidant supply (as air) and cooling, as shown in Fig. 1a. The stack consists of bipolar plates made of FU4369 HT material with a thickness of $5 \mathrm{~mm}$.
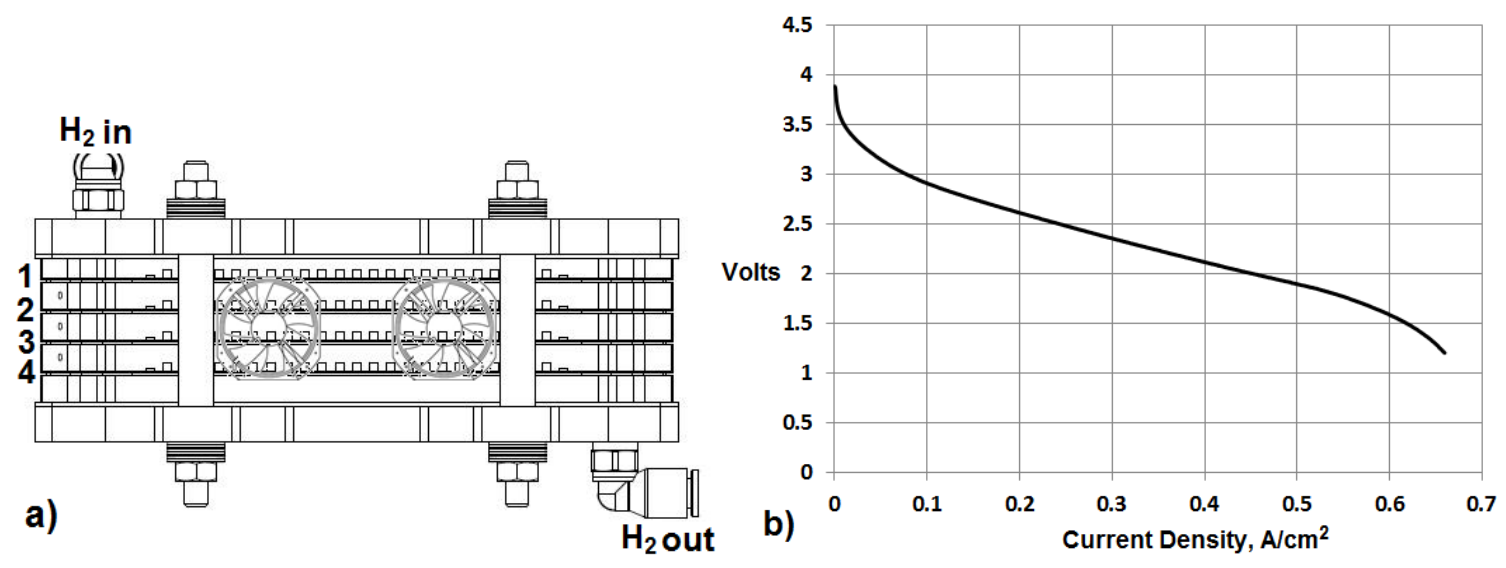

Figure 1. a) Open-cathode fuel cell stack for EIS measurements, 1b) Polarisation curve

High purity hydrogen (99.999 \%) was used during the tests. The fuel cell stack was run in a through flow mode at the anode. Flow rate of hydrogen in the anode was kept constant during all the experiments with a stoichiometry of 2 . The hydrogen supplied was dry. The PEFC stack was operated at ambient temperature $22{ }^{\circ} \mathrm{C}$ and the hydrogen back pressure was held at $0.4 \mathrm{bar}(\mathrm{g})$. Polarisation curves were recorded prior to impedance measurements, as shown in Fig. $1 \mathrm{~b}$.

EIS measurements were carried out through a multichannel frequency response analyzer FRA (Z\#106 WonATech Co). The multichannel FRA consists of five channels and simultaneously measures five impedance spectra through a single induced DC current value. The multichannel system is connected with a RBL488 Dynaload. The EIS measurements were carried out in a galvanostatic mode with a 5\% AC amplitude of the DC current to obtain a linear response from the system at frequencies from $10 \mathrm{kHz}$ to $0.1 \mathrm{~Hz}$. Five channels from the Z\#106 FRA were used to simultaneously measure the impedance of the PEFC stack and the impedance solely for each cell. EIS measurements were carried out at three different current densities 0.1875, 0.3125, and $0.4375 \mathrm{~A} / \mathrm{cm}^{2}$ of the polarisation curve shown in Fig. 1b. The PEFC stack was run over 60 minutes at the required current with no variation in the voltage to ensure a steady state for EIS measurements. EIS measurements for current densities $<0.1875 \mathrm{~A} / \mathrm{cm}^{2}$ were not possible, as the low AC amplitude superimposed onto the DC current made it difficult for the FRA to distinguish between noise and response. At high current densities $>0.4365$ $\mathrm{A} / \mathrm{cm}^{2}$ the stack was not steady for a long period of time due to the high water concentration produced by the oxygen reduction reaction (ORR). 
The resulting stack impedance is shown in a complex plane and represents the electrochemical and diffusion mechanisms in the frequency domain. The diameter of the spectrum decreases from a current density of $0.1875 \mathrm{~A} / \mathrm{cm}^{2}$ to $0.3125 \mathrm{~A} / \mathrm{cm}^{2}$ and increases from a current density of $0.3125 \mathrm{~A} / \mathrm{cm}^{2}$ to $0.4375 \mathrm{~A} / \mathrm{cm}^{2}$, as shown in Fig. 2.

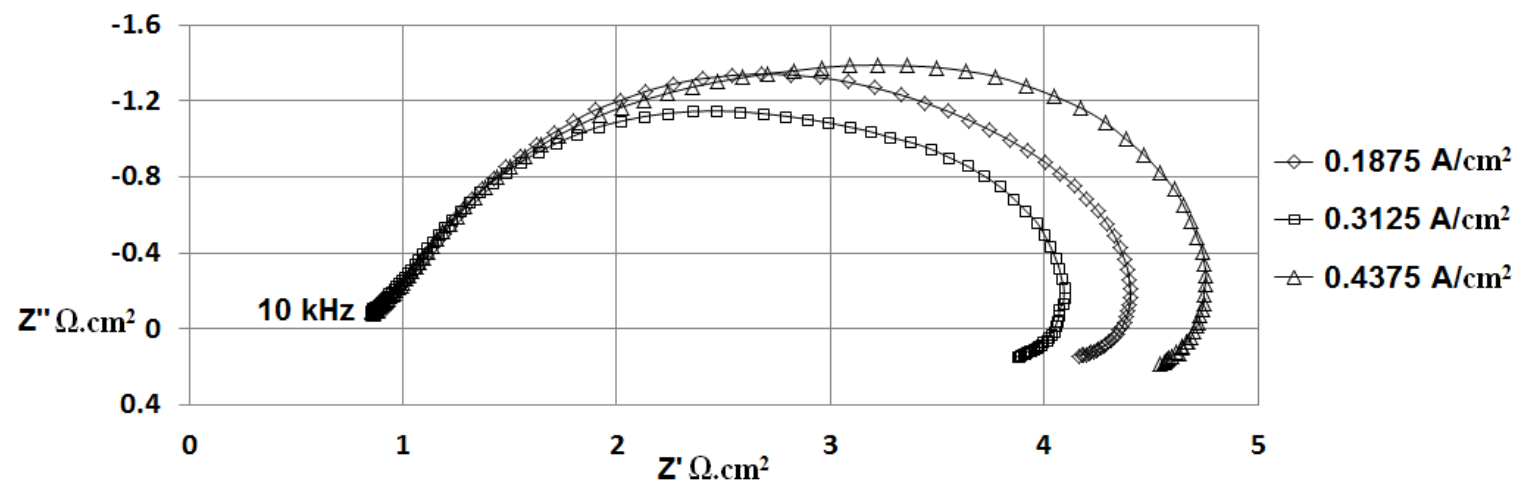

Figure 2. PEFC stack measured impedance data at $0.1875,0.3125$ and $0.4375 \mathrm{~A} / \mathrm{cm}^{2}$

EIS measurements have limitations and present disadvantages as the low impedance values are obscured for low frequencies and some effects are not visible due to a masking effect in the impedance plot (5). Therefore the impedance results of Fig. 2 reflect the overlapping of two semicircles. One at high-medium frequencies is related to the charge transfer resistance during the ORR and decreases with increasing current density, and the other at low frequencies is related to oxygen transport limitations and increases with increasing current density (6). At high frequencies there is no inductive effect (EIS measurements with positive imaginary components Z')') of the measurement cables which deforms the high frequency region of the impedance spectra (7). The sensing cables of the FRA were directly connected to the bipolar plates of the PEFC stack. This allowed the reduction of inductive effects on the EIS measurements at high frequencies by placing the sensing cables from the FRA as far apart as possible from the inductive source. At high frequencies, Fig. 2, it is clearly shown that the $45^{\circ}$ straight line represents the ionic resistance of the catalyst layer as discussed in a previous study (7). At low frequencies inductive effects on the EIS results were apparent for the three current densities. Makharia et al. (8) suggested that the possible reason for this inductive effect at low frequencies are the side reaction and intermediates involved in fuel cell reactions. Roy et al. (9) developed an impedance model to account for the reaction mechanisms that may be responsible for the inductive response at low frequencies, the model proposes the formation of hydrogen peroxide $\left(\mathrm{H}_{2} \mathrm{O}_{2}\right)$ as an intermediate in a two step ORR. It has been reported (10) that crossover of hydrogen to the cathode facilitates the reaction of oxygen and hydrogen at the cathode, generating hydroxyl and hydroperoxyl radicals which react further to produce $\mathrm{H}_{2} \mathrm{O}_{2}$ at the cathode. The hypothesis that $\mathrm{H}_{2} \mathrm{O}_{2}$ may be formed at the cathode of a fuel cell is supported by the results of Inaba et al. (11).

Individual Cell Measurements. The use of the multichannel FRA allows the measurement of the impedance response of each cell of the PEFC stack simultaneously. The cells were numbered from the hydrogen inlet side starting from 1, as shown in Fig.1a. Fig. 3 shows the impedance response for the four cells at $0.1875 \mathrm{~A} / \mathrm{cm}^{2}$. The four 
cells show the inductive effect at low frequencies. There is little difference observed in this inductive effect at low frequencies for the cells at different positions in the stack. The cell located closer to the hydrogen inlet (cell 1) results in a smaller impedance spectrum than the other cells, as shown in Fig. 3. The second cell shows the biggest impedance spectrum which could be related to the structural features of the MEA. The measured data from $10 \mathrm{kHz}$ to $100 \mathrm{~Hz}$ are similar for cells 1,3 and 4 . The difference in impedance response at low frequencies in cells 1,3 and 4 could be related to limitations in oxygen transport through the cathode.

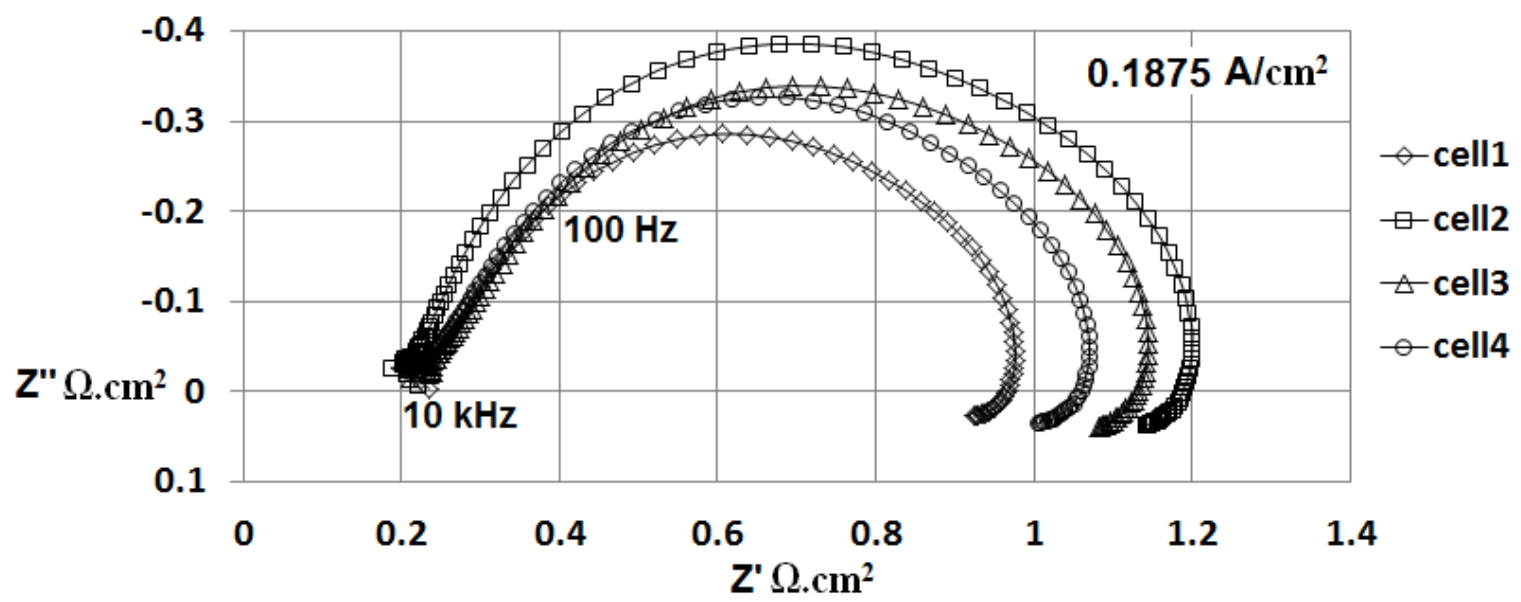

Figure 3. Measured impedance data for different cells of the stack at $0.1875 \mathrm{~A} / \mathrm{cm}^{2}$

Fig. 4 shows the impedance results of the four cells at a current density of 0.3125 $\mathrm{A} / \mathrm{cm}^{2}$. The diameters of the spectra decrease with increasing current density from 0.1875 $\mathrm{A} / \mathrm{cm}^{2}$ to $0.3125 \mathrm{~A} / \mathrm{cm}^{2}$. When the kinetics of the ORR dominate the cell performance such as in the low current density range of the polarisation curve, the impedance spectrum mainly represents the charge transfer resistance during the ORR and its diameter decreases with increasing current density (5). At a current density of 0.3125 $\mathrm{A} / \mathrm{cm}^{2}$ there is an increase in the driving force for the interfacial oxygen reduction process. The impedance spectrum of cell 3 at low frequencies is bigger than cells 1 and 4 . Cell 2 shows the biggest impedance spectrum.

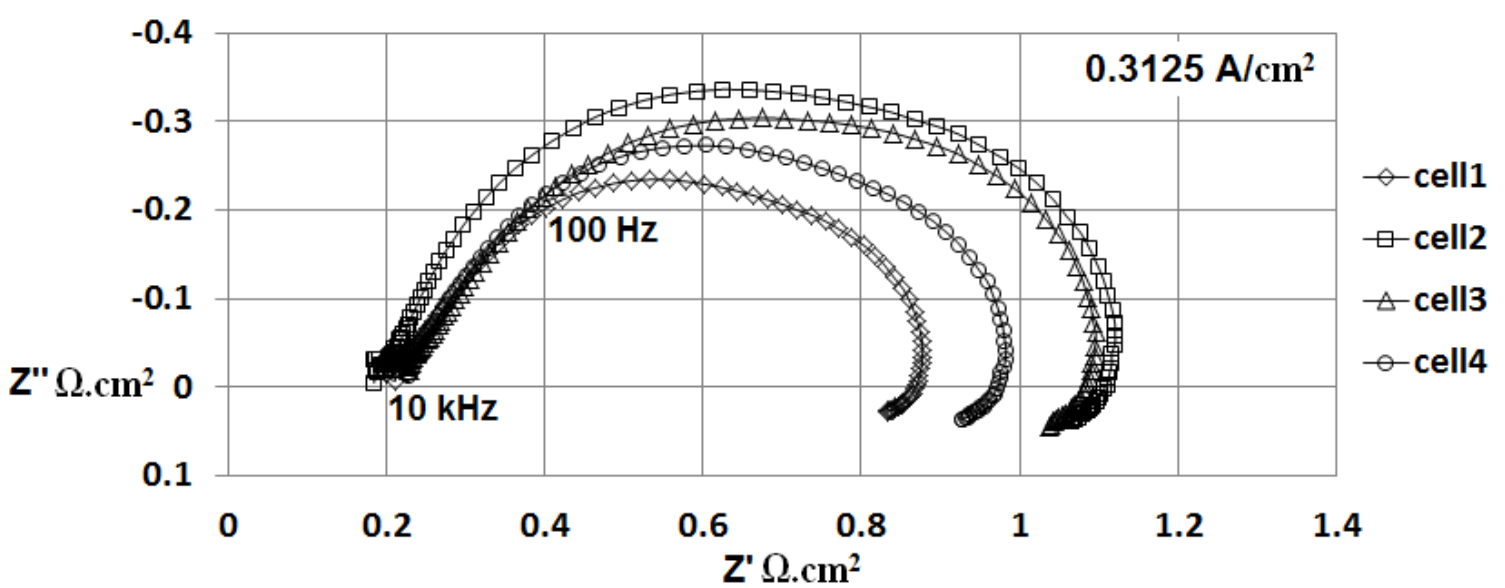

Figure 4. Measured impedance data for different cells of the stack at $0.3125 \mathrm{~A} / \mathrm{cm}^{2}$ 
Fig. 5 shows the impedance results at a current density of $0.4375 \mathrm{~A} / \mathrm{cm}^{2}$. One of the disadvantages of the EIS technique is that multiple energy controlled processes during the electrochemical reaction can be masked in the impedance plot. Therefore in Fig. 5 the semicircles that represent the charge transfer of the ORR and mass transport effects are overlapped in the experimental impedance spectra. Similar results were reported by Yuan et al. (12) when EIS measurements were carried out in a $\mathrm{H}_{2} /$ air PEFC stack. Their results show that EIS measured data feature a single semicircle at high current densities. The increase in diameter of the spectra with increasing current density from $0.3125 \mathrm{~A} / \mathrm{cm}^{2}$ to $0.4375 \mathrm{~A} / \mathrm{cm}^{2}$ as shown in Fig. 5 is attributed to an increase in oxygen transport limitations (6).

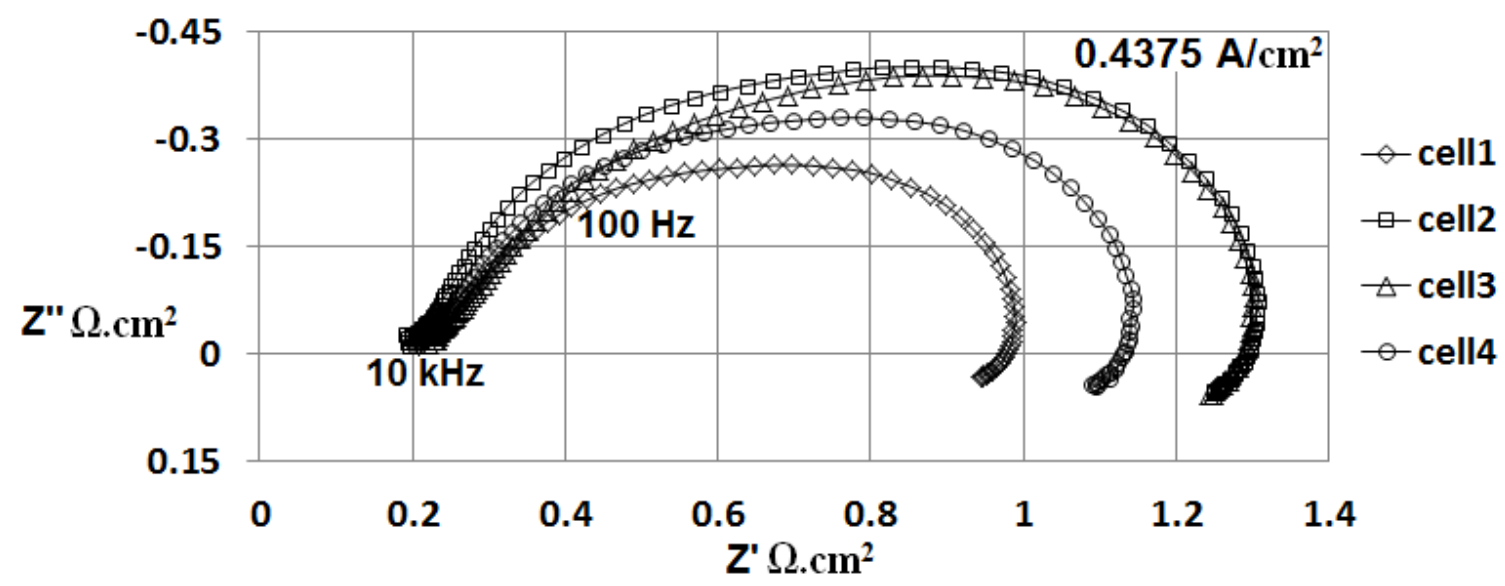

Figure 5. Measured impedance data for different cells of the stack at $0.4375 \mathrm{~A} / \mathrm{cm}^{2}$

\section{Results}

In this study the factors that limit the performance in the open-cathode PEFC stack are assessed through EIS measurements and the details of the impedance model published elsewhere $(3,4)$. It is possible to generate a deeper understanding of the internal phenomenological processes by coupling the experimental EIS technique to the fundamental electrochemical and diffusion theories. The use of equivalent circuits with the experimental EIS technique is a well-established methodology to characterise processes in the PEFC. The electrical circuit that models the PEFC spectrum neglecting anode contribution contains a resistance that accounts for the polymer electrolyte membrane (PEM), GDL and bipolar plate connected in series with a circuit accounting for the cathode catalyst layer (CCL), in which for this specific case is the CCL impedance equation reported in the previous work (4), as such:

$$
Z_{F C}=R_{e}+\frac{\left(R_{C}+Z_{W}\right) \gamma \operatorname{coth}(\gamma x)}{1+Y(i \omega)^{P}\left(R_{C}+Z_{W}\right)}
$$

where

$$
\gamma=\sqrt{R_{p}\left[\frac{1}{R_{C}+Z_{W}}+Y(i \omega)^{P}\right]}
$$


$R_{P}$ is the resistance to the flow of ions in the electrolytic phase of the CCL; $R_{C}$ represents the charge transfer resistance presented in the ORR and is defined as $R_{C}=b / j_{0} \exp \left(\eta_{S} / b\right)$, where $b$ is the Tafel slope, $\eta_{S}$ represents a value of voltage in activation overpotential, and $j_{0}$ is the exchange current; $Z_{W}=R_{W} \tanh \left(i \omega T_{W}\right)^{0.5} /\left(i \omega T_{W}\right)^{0.5}$ is defined as the Warburg impedance and describes diffusion across a finite dimension in the frequency domain (13), with $R_{W}=R T \delta /\left(z^{2} F^{2} c_{0}^{*} D\right)$ defined as resistance for the diffusion process and $T_{W}=\delta^{2} / D$ defined as the time constant to diffuse oxygen through the CCL; $Y$ represents a parameter related to constant phase element (CPE), superscript $P$ represents a parameter to correct the inhomogeneity in the distribution of charge between the electrode-electrolyte inteface; $\omega$ is the angular frequency; $i$ is the imaginary component in impedance; $R_{e}$ represents the total ohmic resistance to flow electrons and ions in the bipolar plate, GDL and PEM.

In the authors' previous study (6) a reference electrode inserted in a PEFC operated with $100 \%$ relative humidity in the anode and a multichannel FRA allowed the separation of the impedance response of the cell and cathode. The results showed that the difference between cell and cathode impedance responses accounted to anode contribution (hydrogen oxidation reaction) becomes negligible at medium and high currents. In this study anode contribution can be considered as negligible for the EIS measurements at medium and high currents. The simulated data from Eq. 1 were compared with the measured EIS data using a Graphic User Interface (GUI) developed in Matlab ${ }^{\circledR}$. The use of the GUI with Eq. 1 for EIS analysis has already been demonstrated in the authors' previous study $(6,7)$. The GUI allows the fitting of the parameters from Eq. 1 to achieve a good agreement between the experimental and simulated data. The least squares fitting method was used in order to find the best-fit between the model and the measured data. A good quality fit is obtained when the sum of the deviations squared (least square error) between the simulated and measured impedance data has a minimum value, for instance $<0.1$. The results show that Eq. 1 cannot reproduce EIS measurements in the positive imaginary part of the complex impedance plane at low frequencies as shown in Fig. 6.

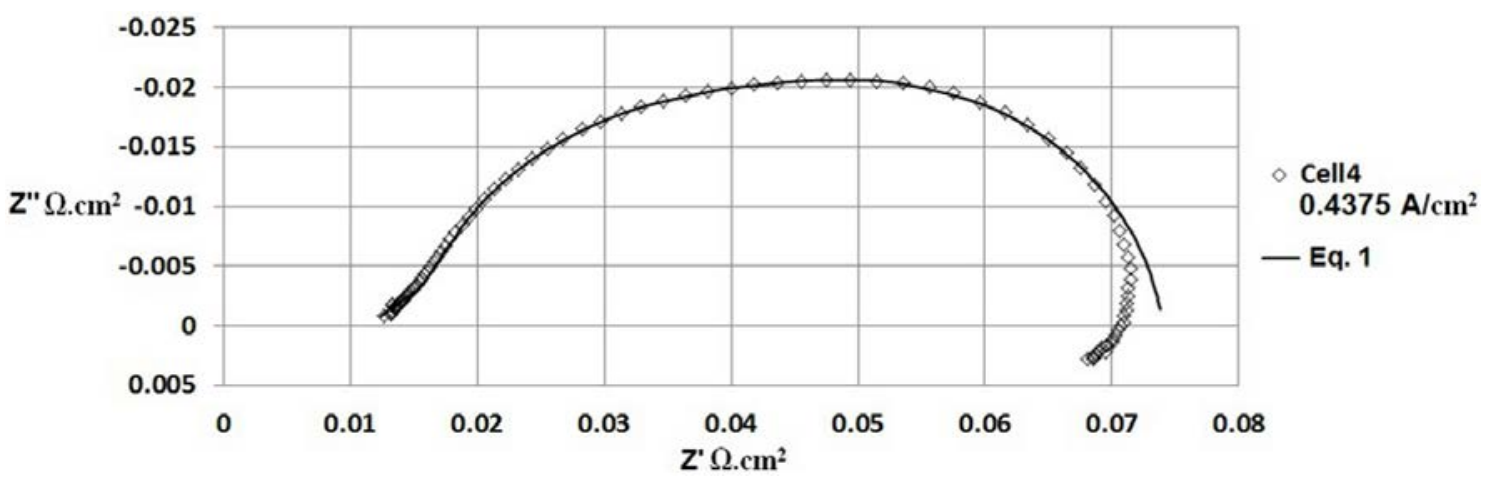

Figure 6. Comparison between measured data and simulated data from Eq.1

\section{Adsorbed Intermediate Species}

EIS measurements in the positive imaginary part of the complex impedance plane at low frequencies are commonly recognised as an inductive loop. This inductive loop has been considered a characteristic of systems containing consecutive heterogeneous reactions with potential-dependent adsorbed intermediate species (14). Ambrosi and Sarli (15) reported that electrochemical reactions that take place within the electrolyte can be 
governed by both the rate constants and the intermediate species adsorbed on the electrode. The authors reported an electrical circuit representing a faradaic process with adsorption, as shown in Fig. 7.

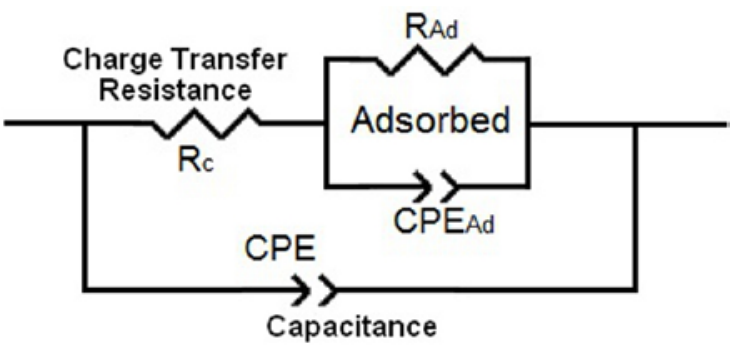

Figure 7. Equivalent electrical circuit representing a faradaic process with adsorption

Fig. 7 shows that if intermediate species are involved in the ORR, an additional equivalent network formed by a pseudo-capacitance $C P E_{A d}$ in parallel with a pseudoresistance $R_{A d}$ should be added in series with the former $R_{C}$ in the $R_{C}-C P E$ combination that represents simple reaction kinetics. As the adsorption only covers a fraction of the total electrode area, such a fraction fluctuates at the same frequency of the potential but differs in phase with it. Ciureanu and Wang (16) reported the same electrical circuit shown in Fig. 7 to account for an electrode with adsorbed intermediate species at the interface between dissimilar materials i.e. nafion-carbon.

The second term on the right-hand side of Eq.1 was derived in previous studies $(3,4)$ by considering simple reaction kinetics $O x+e^{-} \stackrel{K}{\leftrightarrow}$ Re and does not consider adsorbed intermediate species during the ORR which can limit the performance of the PEFC. In Eq. 1 the term expressed as $\left(R_{C}+Z_{W}\right)$ represents the total process resistance during the ORR, charge transfer resistance $R_{C}$ in series with mass transport resistance $Z_{W}$ due to oxygen transport limitations. Bard and Faulkner (17) reported that the overpotential during an electrochemical reaction can be considered as a sum of terms associated with the different reaction steps: mass transport overpotential, charge transfer overpotential and overpotential associated with a preceding reaction. Therefore the electrode reaction can be represented by a resistance composed of a series of resistances representing the various steps during the electrochemical reaction. If the term representing the process associated with the adsorbed intermediate species during the ORR $Z_{A d}=R_{A d} /\left(1+Y_{A_{d}}(i \omega)^{P_{A d}} R_{A d}\right)$ derived from the circuit in Fig. 7 is put in series with $\left(R_{C}+Z_{W}\right)$ in Eq. 1 to consider the total process resistance during the ORR as reported by Bard and Faulkner (17). An approximate representation for the impedance response of the PEFC considering adsorbed intermediate species can be expressed as:

$$
Z_{F C}=R_{e}+\frac{\left(R_{C}+Z_{W}+Z_{A d}\right) \gamma \operatorname{coth}(\gamma x)}{1+Y(i \omega)^{P}\left(R_{C}+Z_{W}+Z_{A d}\right)}
$$

where

$$
\gamma=\sqrt{R_{p}\left[\frac{1}{R_{C}+Z_{W}+Z_{A d}}+Y(i \omega)^{p}\right]}
$$


If the parameters represented in Eq. 3 are fitted to the measured EIS data using the GUI in Matlab ${ }^{\circledR}$ and considering the least square error $<0.1$, it is possible to reproduce the inductive loop at low frequencies as shown in Fig. 8.

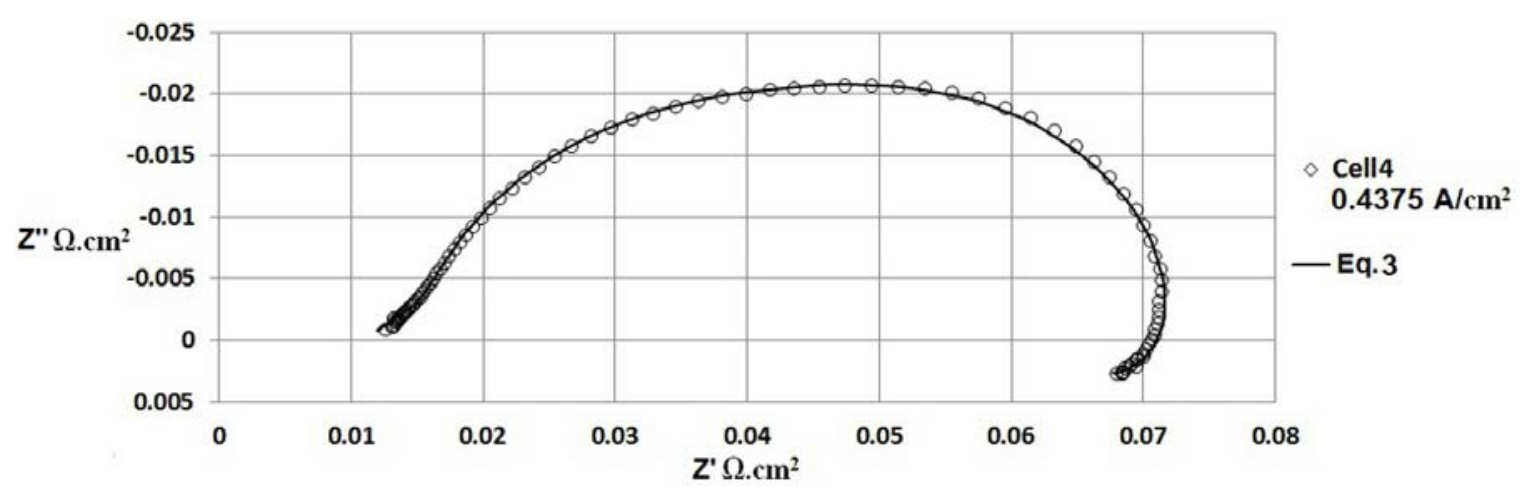

Figure 8. Comparison between measured data and simulated data from Eq. 3

The results show that Eq. 3 is able to reproduce the low frequency loop which is related to adsorbed intermediate species in the ORR. The electrochemical and diffusion mechanisms of the measured data were calculated through Eq. 3 and the results are shown in Tab. I. The parameter $R_{\text {Ad }}$ that accounts for the adsorbed intermediate species during the ORR resulted in a negative number and became more negative with increasing current density for all cells, as shown in Tab. I. Bai and Conway (18) demonstrated in their work that the inductive loop at low frequencies in electrochemical systems with adsorbed intermediate species is related to a negative value of adsorbed species coverage $\varphi$ depending on potential $(d \varphi / d E)$. Ciureanu and Wang (16) reported EIS measurements in a $\mathrm{H}_{2} / \mathrm{H}_{2}+\mathrm{CO}$ PEFC. EIS measurements showed the inductive loop at low frequencies with increasing bias potential. In their study the electrical circuit shown in Fig. 7 was applied with EIS measurements and this resulted in negative values for $R_{A d}$. They concluded that the negative values in $R_{A d}$ are a result of the decrease of CO coverage on the electrode with increasing bias potential due to oxidation of CO by oxygenated species on the electrode-electrolyte interface. The process responsible for the inductive characteristic at low frequencies shown in Fig. 8 could be attributed to hydrogen crossover through the PEM or bipolar plates to form $\mathrm{H}_{2} \mathrm{O}_{2}$ during the ORR as reported by Roy et al. (9). A further study to consider crossover of hydrogen through bipolar plates made of FU4369 HT material should be investigated.

Charge Transfer Resistance. The measured data from $10 \mathrm{kHz}$ to $100 \mathrm{~Hz}$ are similar for cells 1, 3 and 4, as shown in Figs. 3, 4 and 5. The temperature distribution through this particular stack is not very significant in the kinetics of the electrochemical reaction as the measured data in the frequency range of $10 \mathrm{kHz}-100 \mathrm{~Hz}$ presented the same trend at the same current density with an exception for cell 2. The parameter charge transfer resistance $R_{C}$ calculated from EIS measurements and Eq. 3 is shown in Tab. I. The charge transfer resistance $R_{C}$, whose dependence on electrode potential is given by the Tafel equation and reflects the increase in the driving force during the ORR, remains constant for cells 1,3 and 4 at the same current density. $R_{C}$ is a function of the exchange current density $j_{0}$, as such $R_{C}=b / j_{0} \exp \left(\eta_{s} / b\right)$. A decrease in $j_{0}$ results in an increase of $R_{C}$ and thus a decrease in the activity of the electrode surface is produced. Cell 2 shows a higher $R_{C}$ than the rest of the cells at the same current density which can be attributed to low 
electrocatalytic activity in cell 2. Therefore the activation overpotential in the PEFC stack is dominated by the charge transfer resistance of cell 2 . As expected $R_{C}$ decreases in the four cells with increasing current density.

TABLE I. Charge transfer resistance $R_{C}$, mass transport resistance $R_{W}$ and adsorption resistance $R_{A d}$ calculated from Eq. 3

\begin{tabular}{|c|c|c|c|}
\hline & Rc $\Omega . \mathrm{cm}^{2}$ & $\boldsymbol{R}_{w} \Omega . \mathrm{cm}^{2}$ & $R_{A d} \Omega . \mathrm{cm}^{2}$ \\
\hline \multicolumn{4}{|l|}{$0.1875 \mathrm{~A} / \mathrm{cm}^{2}$} \\
\hline Cell1 & 0.7712 & 0.0128 & -0.128 \\
\hline Cell2 & 0.9312 & 0.1216 & -0.192 \\
\hline Cell3 & 0.7712 & 0.1584 & -0.176 \\
\hline Cell4 & 0.7712 & 0.0736 & -0.16 \\
\hline \multicolumn{4}{|l|}{$0.3125 \mathrm{~A} / \mathrm{cm}^{2}$} \\
\hline Cell1 & 0.6544 & 0.064 & -0.136 \\
\hline Cell2 & 0.8144 & 0.176 & -0.184 \\
\hline Cell3 & 0.6544 & 0.2304 & -0.2 \\
\hline Cell4 & 0.6544 & 0.152 & -0.184 \\
\hline \multicolumn{4}{|l|}{$0.4375 \mathrm{~A} / \mathrm{cm}^{2}$} \\
\hline Cell1 & 0.6336 & 0.1952 & -0.184 \\
\hline Cell2 & 0.7568 & 0.4272 & -0.232 \\
\hline Cell3 & 0.6336 & 0.504 & -0.328 \\
\hline Cell4 & 0.6336 & 0.3552 & -0.248 \\
\hline
\end{tabular}

Ohmic Resistance. Fig. 9 shows the high frequency region of the EIS measurements at the three different current densities. The measured data of cell 2 are not shown in Fig. 9 as the data of this cell from $10 \mathrm{kHz}$ to $100 \mathrm{~Hz}$ showed a different trend compared to the rest of the cells as shown in Figs. 3, 4 and 5. Cell 3 shows a slightly higher ohmic resistance than cell 1 and 4 at the three current densities, this effect can be noticeable in the real part Z' at $10 \mathrm{kHz}$ of the measured data, as shown in Fig. 9. The increase in ohmic resistance could be attributed to dehydration in the PEM which may have been caused by an increase in reaction generated heat in cell 3. Ohmic resistance seems to be more sensitive than charge transfer resistance to the temperature distribution through the stack.
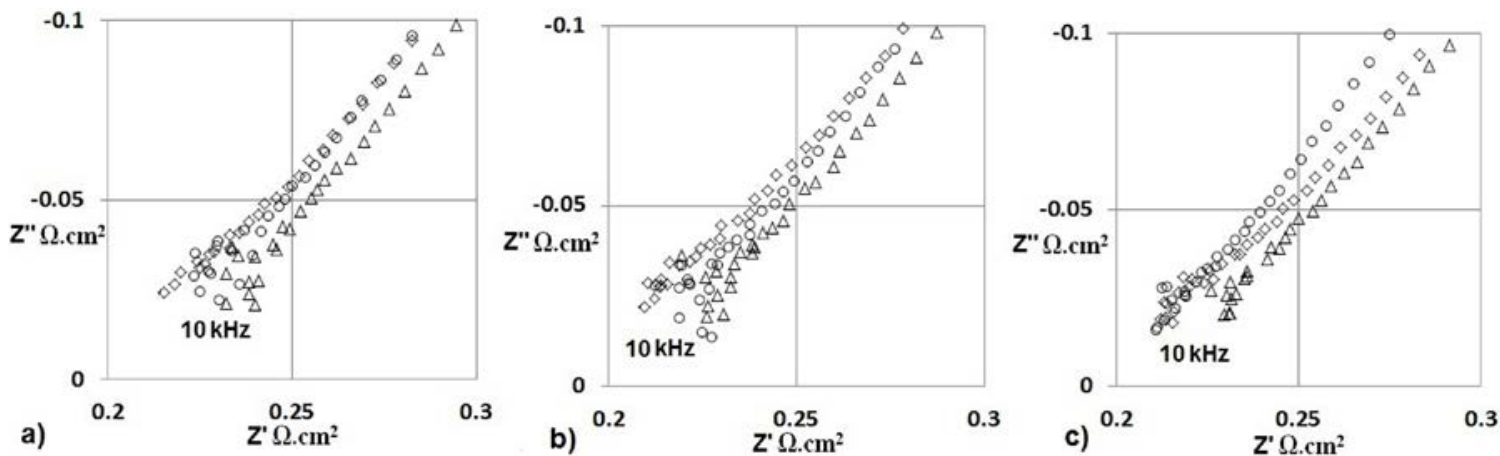

Figure 9. High frequency EIS measurements for cell $1 \diamond$, cell $3 \Delta$ and cell $4 \circ$; a) $0.1875 \mathrm{~A} / \mathrm{cm}^{2}$, b) $0.3125 \mathrm{~A} / \mathrm{cm}^{2}$, c) $0.4375 \mathrm{~A} / \mathrm{cm}^{2}$

Oxygen Transport Limitations. The mass transport resistance $R_{W}$, due to gaseous oxygen transport limitations and as a function of the gaseous oxygen equilibrium concentration in the CCL-GDL interface, increases with increasing current density for all cells, as shown in Tab. I. Cells 2 and 3 show higher mass transport resistance than cells 1 and 4. Cell 3 shows the highest mass transport resistance as shown in Fig. 10. The 
increase in mass transport resistance of cell 3 could be attributed to high water concentration produced by the ORR which affects the transport of oxygen to reach the reaction sites in the CCL; however the increase in ohmic resistance (PEM dehydration) in cell 3, shown in Fig. 9, demonstrates a contrasting case.

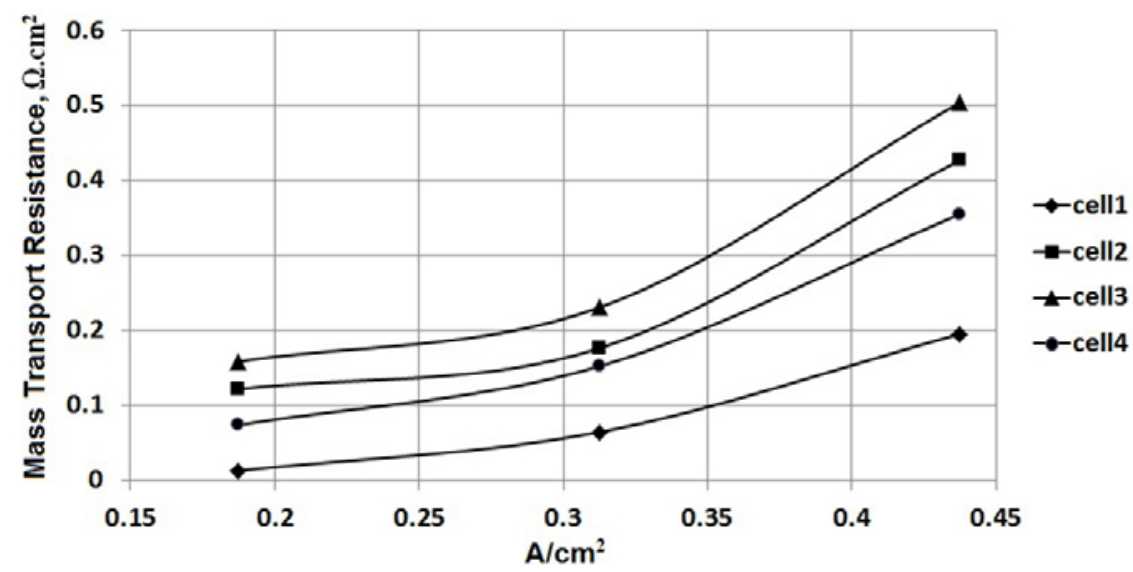

Figure 10. Estimation of mass transport resistance in each individual cell

Santa Rosa et al. (19) studied the effect of critical conditions on an open-cathode 8cell stack operated at ambient temperature and pressure. Three different fans for oxidant supply and cooling were used to investigate how the air flow rate affects the stack performance. A $5 \mathrm{~V}$ DC fan was found to be the best option because it ensures enough stack cooling and oxidant supply. The authors concluded that a fan with lower voltage affects the stack performance at medium-high currents because lower air fan flow rate cannot expel excessive reaction generated heat which in turns leads to dehydration in the PEM (high ohmic resistance). In this study, simultaneous EIS measurements demonstrate that inhomogeneity of air flow rate in an open-cathode PEFC stack not only leads to an increase in ohmic resistance but also leads to an increase in oxygen transport limitations in the cells. Limitations in air flow rate decrease the gaseous oxygen equilibrium concentration in the GDL-CCL interface and increase mass transport resistance. The position and distance of the fans with respect to the open cathodes in this particular stack played an important role for oxygen transport limitations. Two 5V DC axial fans were centred $1 \mathrm{~cm}$ over the open cathode of cell 3 as shown in Fig. 1a. Air enters the fan evenly as it follows the physics of moving from a high pressure area into a low pressure area. The air then spreads outwards as it leaves the fan due to a centrifugal force. This results in a vacuum area absent of air flow in the centre of the fan and therefore the majority of the airflow is around the sides of the fan. A fan characteristic curve (FCC) presents three typical regions for axial fans: a stalling, an unstable and an optimal operating region (20). Sasmito et al. (20) reported that the interception between the operating point of an OpenCathode fuel cell stack and a FCC should be located in the optimal operating region and be sufficiently far away from any unstable and stalling region. With most fans there is no air flow right in the centre of a fan. The majority of the airflow is around the sides of the fan; this effect limits the pressure within the open cathode in cell 3 and thus the operating point of cell 3 corresponds to the stalling point in the FCC. This effect could explain the increase in oxygen transport limitations and the increase in reaction generated heat in cell 3 which increases the ohmic resistance. 


\section{Discussion}

The inhomogeneous performance along a PEFC stack can be monitored with the use of simultaneous EIS measurements. One of the disadvantages of the EIS technique is that multiple energy controlled processes during the electrochemical reaction can be masked in the impedance measurements. The interpretation of the electrochemical and diffusion mechanisms which are truly happening in a PEFC stack can be unveiled by relating the electrochemical impedance data to the fundamental theory of PEFCs. It is possible to diagnose degradation in MEAs which yield low electrochemical activity in the electrodes through experimental EIS and modelling. Oxygen transport limitations due to flooding can be diagnosed at low frequencies of EIS measurements. EIS measurements can also provide a direct correlation about variations in air flow through the open cathodes in an open-cathode PEFC. Finally another common effect limiting the stack performance is the presence of adsorbed intermediate species during the ORR. This effect has been identified in EIS measurements as an inductive loop at low frequencies. Open-cathode PEFC stacks are exposed to a range of atmospheric compositions which can include sulphur dioxide, nitrogen oxides, ionic contamination, carbon monoxide, etc. This level of understanding is critical to industry to drive the development, optimisation and running of PEFCs as reliable commercial products. A further investigation to include poisoning effect on open-cathode PEFCs which is normally represented in the positive imaginary part of the complex impedance plane at low frequencies will be researched in future work.

\section{Conclusions}

This study has demonstrated that simultaneous EIS is a powerful tool for in-situ diagnosis of a PEFC stack. EIS measurements that account for each cell in an opencathode PEFC stack were compared with simulated data from the model reported in the authors' previous study $(3,4)$. The model was modified to include adsorbed intermediate species mechanisms during the ORR and the results showed a good agreement between measured data and simulated data. Electrochemical and diffusion mechanisms for each cell in the PEFC stack were calculated through EIS measurements and the model. The results showed that the activation overpotential in the whole PEFC stack is dominated by low electrocatalytic activity in one of the cells. The results also showed that oxygen transport limitations and ohmic resistance are attributed to variations in flow of the air through the open cathodes in the stack. The results also revealed that adsorbed species during the ORR limit the performance of the PEFC stack. Further experiments to account for the exact intermediate species should be carried out. This newly established EIS knowledge will enable an assessment of the state of health of operational fuel cell stacks.

\section{Acknowledgements}

The authors thank the Mexican National Council for Science and Technology (CONACYT) for the sponsorship of the Ph.D. research study of S. Cruz-Manzo (Grant no. 183195). 


\section{References}

1. R. Mohtadi, W. K. Lee, and J. W. V. Zee, J. Power Sources, 138, 216 (2004).

2. J. M. Moore, P. L. Adcock, J. B. Lakeman, and G. O. Mopsted, J. Power Sources, 85, 254 (2000).

3. S. Cruz-Manzo, P. Rama, and R. Chen, J. Electrochem. Soc., 157, B400 (2010).

4. S. Cruz-Manzo, P. Rama, and R. Chen, J. Electrochem. Soc., 157, B1865 (2010).

5. V. A. Paganin, C. L. F. Oliveira, E. A. Ticianelli, T. E. Springer and E. R. Gonzalez, Electrochim. Acta, 43, 3761 (1998).

6. S. Cruz-Manzo, R. Chen and P. Rama, Int. J. Hydrogen Energy, 38, 1702 (2013).

7. S. Cruz-Manzo, R. Chen and P. Rama, J. Fuel Cell Science Technology, 9, 051002 (2012).

8. R. Makharia, M. F. Mathias and D. R. Baker, J. Electrochem. Soc., 152, A970 (2005).

9. S. K. Roy, M. E. Orazem and B. Tribollet, J. Electrochem. Soc., 154, B1378 (2007).

10. J. Xie, D. L. Wood III, K. L. More, P. Atanassov, and R. L. Borup, J. Electrochem. Soc., 152, A1011 (2005).

11. M. Inaba, H. Yamada, J. Tokunaga, and A. Tasaka, Electrochem. Solid-State Lett., 7, A474 (2004).

12. X. Yuan, J. C. Sun, H. Wang and J. Zhang, J. Power Sources, 161, 929 (2006).

13. N. Fouquet, C. Doulet, C. Nouillant, G. Dauphin-Tanguy and B. OuldBouamama, J. Power Sources, 159, 905 (2006).

14. M. Keddam, O. R. Mottos, and H. Takenouti, J. Electrochem. Soc., 128, 257 (1981).

15. V. Ambrosi and A. Di Sarli, Anti-Corrosion, 40, 4 (1993).

16. M. Ciureanu and H. Wang, J. Electrochem. Soc., 146, 4031 (1999).

17. A. J. Bard and L. R. Faulkner, Electrochemical Methods, p. 24, John Wiley \& Sons Inc., New York (2001).

18. L. Bai and B. E. Conway, J. Electrochem. Soc., 137, 3737 (1990).

19. D.T. Santa Rosa, D.G. Pinto, V.S. Silva, R.A. Silva, and C.M. Rangel, Int. J. Hydrogen Energy, 32, 4350 (2007).

20. P. Sasmito, E. Birgersson, K. W. Lum, A. S. Mujumdar, Renewable Energy, 37, 325 (2012). 